Check for updates

Cite this: RSC Adv., 2018, 8, 24297

\title{
Biomimetic fabrication of superhydrophobic loofah sponge: robust for highly efficient oil-water separation in harsh environments $\uparrow$
}

\author{
Mingguang Yu, (D) ${ }^{a}$ Binbin Lin, ${ }^{a}$ Shangxian Chen, ${ }^{a}$ Qianjun Deng, ${ }^{a}$ Guang Liu ${ }^{c}$ \\ and Qing Wang*b
}

Oil/water separation has become an increasingly important field due to frequent industrial oily wastewater emission and crude oil spill accidents. Herein, we fabricate a robust superhydrophobic loofah sponge via a versatile, environmentally friendly, and low-cost dip coating strategy, which involves the modification of commercial loofah sponge with waterborne polyurea and fused $\mathrm{SiO}_{2}$ nanoparticles without the modification of any toxic low-surface-energy compound. The as-prepared loofah sponge showed excellent superhydrophobic/superoleophilic properties and exhibited robustness for effective oil-water separation in extremely harsh environments (such as $1 \mathrm{M} \mathrm{HCl}, 1 \mathrm{M} \mathrm{NaOH}$, saturated $\mathrm{NaCl}$ solution and hot water higher than $95^{\circ} \mathrm{C}$ ) due to the remarkably high chemical stability. In addition, the as-prepared loofah sponge was capable of excellent anti-fouling, has self-cleaning ability and could act as the absorber for effective separation of surfactant-free oil-in-water emulsions. More importantly, the asprepared loofah sponge demonstrated remarkable robustness against strong sandpaper abrasion and finger wipes, while retaining its superhydrophobicity and efficient oil/water separation efficiency even after more than 50 abrasion cycles. This facile and green synthesis approach presented here has the advantage of large-scale fabrication of a multifunctional biomass-based adsorbent material as a promising candidate in anti-fouling, self-cleaning, and versatile water-oil separation.

Received 22nd May 2018

Accepted 5th June 2018

DOI: $10.1039 / \mathrm{c} 8 \mathrm{ra04336a}$

rsc.li/rsc-advances
Recently, a variety of functional materials for oil/water separation were fabricated through the rational control of surface structures and chemical compositions to possess the property of superhydrophobicity/superoleophilicity, ${ }^{14-21}$ since Jiang $^{22}$ et al. first discovered the oil/water separation materials inspired by special wettability in 2004. Li et al..$^{23}$ demonstrated a superhydrophobic polyurethane sponge fabricated by coating $\mathrm{TiO}_{2}$ nanoparticles and a low-surface-energy compound (octadecanoic acid) onto its skeleton surface. The coated PU sponges via the ultrasonic-assisted dip coating (UADC) method showed excellent oil-water separation ability in extremely high acidic and alkaline environments as well as the extremely high salt concentration of seawater. Zhang et al. ${ }^{24}$ demonstrated a facile salt-induced phase-inversion method to fabricated superhydrophilic and underwater superoleophobic PAA-g PVDF membranes for separation of oil-in-water emulsions with high separation efficiency and much higher fluxes. Li et al. ${ }^{25}$ developed a facile approach to fabricate superhydrophobic bags filled with pristine PU sponges for highly efficient oil/water separation in harsh environments.

For super-wetting materials, the fragile micro-nano surface roughness structure is so vulnerable that it can fail easily under the influence of external mechanical forces or friction. This is becoming the biggest bottleneck that limits large-scale industrial application in the area of oil-water separation. ${ }^{26}$ In order to 
solve this problem and improve the mechanical properties of the surface of superhydrophobic materials, many methods have been proposed..$^{27-38} \mathrm{Lu}$ et al. $^{27}$ used a perfluorosilane-coated titanium dioxide nanoparticles ethanolic suspension as a paint to fabricate micro-nano multi-level structure, with commercial adhesives as a binding layer to improve the robustness and abrasion resistance, to construct robust superhydrophobic surfaces on several substrates, which maintained excellent superhydrophobicity even after finger-wipe, knifescratch, and sandpaper abrasion. Chen et al. ${ }^{30}$ successfully fabricated superhydrophobic surfaces with remarkable robustness against knife scratch and sandpaper abrasion on various substrates in a similar method called "Paint + Adhesive". Tricoli et $a{ }^{32}$ reported a novel synthesis method to construct ultradurable superhydrophobic surfaces by using a PU-PMMA colloidal suspension and fluoro-functionalized nanostructured silica $\left(\mathrm{F}-\mathrm{SiO}_{2}\right)$. After curing, the highly transparent and excellent superhydrophobic surfaces with hierarchical micro-nano structure and ultralow surface energy were formed, which presented outstanding mechanical, chemical, and photo durability, and retained their water repellency even after 250 rotary abrasion cycles. However, the fluorocarbon reagents used were expensive and environmentally incompatible, which largely limited their applications. Consequently, robust and environmentally friendly approaches for construction of fluorine-free, green superhydrophobic absorbent materials are highly desirable.

With improving environmental protection and a growing shortage of oil resources, the use of renewable natural materials is becoming increasingly attractive because of their high absorption capacities as well as renewability and biodegradability. ${ }^{39-54}$ Yang et al. ${ }^{39}$ prepared a novel diisocyanate-modified lignin xerogel using renewable lignin as the precursor via a solgel process and ambient pressure drying method. The asprepared xerogel showed high performance in self-cleaning and superhydrophobicity without further hydrophobic modification. Zhou et al. $^{\mathbf{4 6}}$ fabricated superhydrophobic microfibrillated cellulose aerogels with high lipophilicity, ultralow density, superior porosity as well as extremely high mechanical stability. The aerogels exhibited excellent oil/water selective absorption capacity and superior reusability. However, the relatively complicated operation procedures and low structural stability have limited the actual applications for oil/water separation.

Loofah sponges are available at very low cost and are popular in China and Southeast Asia. The loofah sponge possesses a continuous 3D macroporous surface with a strong rigid structure that is similar to those of polymer-based sponges. Loofah sponge has many applications in life, such as a filter material for filtration and purification, kitchen cleaning supplies and decorative materials, due to its good water absorption capacity and stable structure. Meanwhile, it can also be used as a kind of fuel for cooking in remote rural areas. In this study, we fabricated a 3D macroporous oil-absorbing material prepared from a fibrous loofah sponge via a facile and efficient dip coating process. The fumed nano silica and waterborne polyurea adhesive were first dispersed into ethanol, then the loofah sponge was introduced into the above solution. After curing at $60{ }^{\circ} \mathrm{C}$, the superhydrophobic/superoleophilic biomass-based loofah sponges have been obtained. The asprepared loofah sponges showed excellent superhydrophobic/ superoleophilic properties and exhibited robustness for effective oil-water separation. In this work, the fumed $\mathrm{SiO}_{2}$ nanoparticles not only enhance the surface roughness of the loofah sponge but also act as a low-surface-energy compound material, which endow the sponge with excellent superhydrophobic properties. Most importantly, due to the excellent adhesion of the polyurea adhesive, it can tightly bond $\mathrm{SiO}_{2}$ nanoparticles to the loofah sponge and endow the sponge with superior chemical stability and robustness for oil-water separation under harsh environments, including strong acidic, alkaline, and saturated salt aqueous solutions, hot water, mechanical abrasion and finger abrasion. These extraordinary properties suggest that the as-prepared loofah sponge will be a promising candidate for large-scale oil-water separation.

\section{Experimental}

\section{Materials and methods}

Loofah sponge was obtained locally. Waterborne polyurea adhesive (WPU) was purchased from Qingdao jinwanli chemical co. LTD. Fumed nano silica $(\sim 25 \mathrm{~nm})$ was purchased from Sigma-Aldrich (Shanghai, China). Kerosene was purchased from Macklin (Shanghai, China). Ethanol, hexane and chloroform were purchased from Guangzhou Chemical Reagent Co. (Guangzhou, China). Deionized water was made in-house. All the chemicals were used as received without any further purification.

\section{Pretreatment of the loofah sponge}

Raw loofah sponge was cut into smaller-sized pieces, and then washed several times with deionized water to remove dust. Subsequently, the loofah sponge was put into $1 \mathrm{M} \mathrm{NaOH}$ aqueous solution and stirred mechanically for $2 \mathrm{~h}$. After alkali treatment, the loofah sponge was washed several times with deionized water to remove $\mathrm{NaOH}$, then the pre-treated loofah sponge was dried under vacuum at $50{ }^{\circ} \mathrm{C}$ for $12 \mathrm{~h}$.

\section{Preparation of silica@PU coated loofah sponge}

In a typical preparing process, $2 \mathrm{~g}$ of fumed silica nanoparticles and $1 \mathrm{~g}$ of water-based polyurea adhesive were added into $100 \mathrm{~mL}$ of ethanol with ultrasonic dispersion for at least $2 \mathrm{~h}$ to obtain a stable milky white colloidal suspension. The pretreated loofah sponges were dipped into the suspension for $2 \mathrm{~h}$ under vigorous mechanical stirring. Then, the loofah sponge was dried in an oven at $60^{\circ} \mathrm{C}$ for at least $12 \mathrm{~h}$ to obtain the stable water-repellent loofah sponge.

\section{Characterization}

Structures of the raw loofah sponge and as-prepared sponge were analysed by Fourier transform infrared (FT-IR) spectroscopy on a Spectrum 2000 FT-IR spectrometer (Perkin Elmer). The elemental composition of raw loofah and as-prepared 
samples were determined using X-ray photoelectron spectroscopy (XPS) on a Thermo Electron Escalab 250 spectrometer with $\mathrm{Li} \mathrm{K} \alpha$ radiation $(20 \mathrm{eV})$ as the exciting source. The morphology and elemental mapping distribution of the sample were observed on a thermal field-emission scanning electron microscope (FESEM: Quanta 400F, FEI) at $15 \mathrm{kV}$. Water contact angle measurements (WCAs) were measured on a Dataphysics OCA20 Contact-Angle System with liquid droplets of $5 \mu \mathrm{L}$. The reported values are the averages of measurements in least 5 different positions.

\section{Results and discussion}

The one-step, rapid and cost-effective fabrication process for the superhydrophobic/superoleophilic loofah sponge is illustrated in Scheme 1. The fused nano-SiO ${ }_{2}$ and waterborne polyurea adhesive were added in an absolute ethanol solution under ultrasonication for $2 \mathrm{~h}$ to obtain a homogeneous paint-like suspension. An economical and biodegradable loofah sponge with a three-dimensional hierarchical porous structure was used as the frame for coating. After pre-treatment with $1 \mathrm{M}$ $\mathrm{NaOH}$ solution to remove the existence of the natural plant wax layer and the lignin, the cleaned pristine loofah sponge was dipped into the above homogeneous suspension for $2 \mathrm{~h}$ under vigorous mechanical stirring. Then, the as-prepared loofah sponge was dried at $60^{\circ} \mathrm{C}$ for $12 \mathrm{~h}$ to obtain the stable waterrepellent loofah sponge. Taking advantage of the powerful stirring effects, the polyurea molecules and $\mathrm{SiO}_{2}$ nanoparticles diffused quickly into the loofah sponge frame. The urethane bond in the polyurea molecules formed van der Waals force interactions with the hydroxyl groups on the loofah fibers. After curing, the polyurea and the fumed nano silica were tightly attached onto the loofah sponge, which provide the loofah sponge with excellent superhydrophobicity and chemical stability for use as an adsorbent in practical oil-water separation applications in harsh environments.

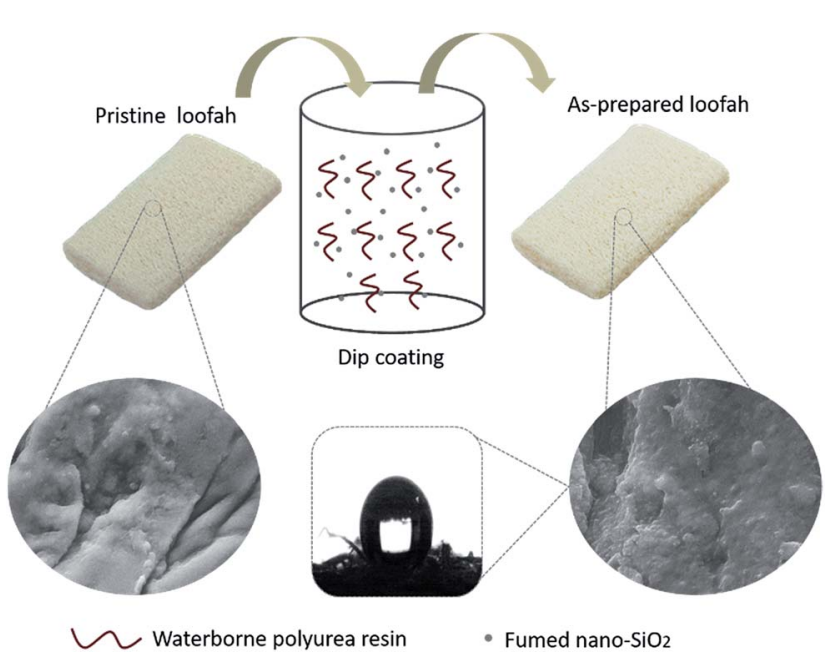

Scheme 1 Schematic illustration of the fabrication of the superhydrophobic loofah sponge.
The surface chemical compositions of pristine loofah sponge, WPU modified loofah sponge and silica@WPU modified loofah sponge were determined by Fourier transform infrared spectroscopy (FTIR). As shown in Fig. 1a, comparing with the spectrum of pristine loofah, the $\mathrm{C}=\mathrm{O}$ stretching vibration peak at about $1732 \mathrm{~cm}^{-1}$ showed a noticeable increase in intensity on the surface of WPU modified loofah, indicating that a considerable number of urethane groups were introduced after WPU modification. The peak at about $1509 \mathrm{~cm}^{-1}$ was attributed to the deformation vibration of $\mathrm{N}-\mathrm{H}$ in urethane, which was a little weaker than that of $\mathrm{C}=\mathrm{O}$ of $1732 \mathrm{~cm}^{-1}$ due to the stretching vibration of $\mathrm{N}-\mathrm{H}$. The stretching vibration near the $1066 \mathrm{~cm}^{-1}$ absorption peak was attributed to the ether bond and ammonia ester bond of $\mathrm{C}-\mathrm{O}-\mathrm{C}$. The infrared spectra analysis proved that polyurea adhesive was successfully grafted onto the loofah fiber surface. In addition, the characteristic peak at $465 \mathrm{~cm}^{-1}$ belonged to the bending vibration peak of $\mathrm{Si}-\mathrm{O}-\mathrm{Si}$. These observations indicated that fumed nano silica and WPU were included on the loofah sponge surface, which confirmed successful synthesis of $\mathrm{SiO}_{2} @ W P U$ modified loofah sponge.

To further prove the above results, X-ray photoelectron spectroscopy (XPS) and X-ray energy dispersive spectroscopy (EDS) were employed to determine the surface elemental compositions of the pristine loofah sponge and the as-prepared loofah sponge. The values for the concentration of the loofah
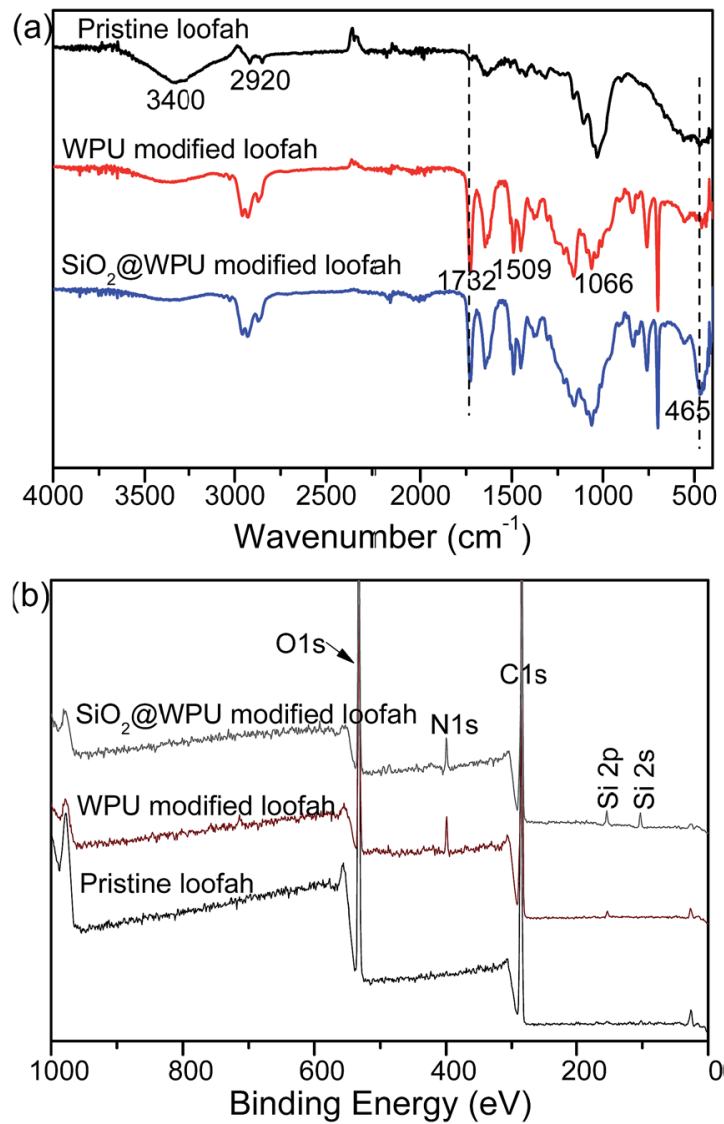

Fig. 1 FT-IR spectra (a) and XPS spectra (b) of pristine loofah, WPU modified loofah, and $\mathrm{SiO}_{2}$ aWPU modified loofah. 
surface chemical elements can be estimated (demonstrated in Table S1 of the ESI †). As depicted in Fig. 1b, the XPS spectrum of original loofah was composed of the elements carbon $(286.5 \mathrm{eV})$ and oxygen $(533.0 \mathrm{eV})$, which can be inferred due to the lignocellulosic characteristic of vegetable materials. In the WPU modified loofah sponge, the relative atomic concentration of carbon increased from $59.75 \%$ to $77.24 \%$, the relative atomic concentration of oxygen decreased from $40.25 \%$ to $18.78 \%$, a new peak with binding energy of $407.3 \mathrm{eV}(\mathrm{N}$ 1s) appeared, strongly confirming that polyurea was successfully attached onto the loofah surface. For the as-prepared loofah sponge, two new peaks with binding energies of $154.23 \mathrm{eV}$ ( $\mathrm{Si} 2 \mathrm{~s}$ ) and $102.14 \mathrm{eV}$ (Si 2p) appeared, which strongly demonstrated the loofah sponge was fully covered by fumed silica particles and WPU adhesive. In addition, elemental mapping analysis based on X-ray energy dispersive spectroscopy as shown in Fig. 2 also confirmed that the as-prepared loofah sponge was successfully coated with polyurea and fused $\mathrm{SiO}_{2}$ nanoparticles.

The typical scanning electron microscopy (SEM) images of the pristine loofah sponge and as-prepared loofah sponge with different magnifications, which were used to obtain the surface morphological changes in the loofah sponge after dip casting, are shown in Fig. 3. As shown in Fig. 3a1, the pristine loofah sponge had a three-dimensional hierarchical porous structure. The magnified SEM images (Fig. 3a2 and a3) revealed that the loofah skeleton surface was slightly rough with several shallow channels and small folds. This unique structure of loofah sponge provided a larger surface area which could be beneficial to load more fused silica nanoparticles on the surface of the loofah fibers. As depicted in Fig. 3b, after dip casting with a homogeneous ethanol suspension composed of fused nano$\mathrm{SiO}_{2}$ and WPU adhesive, the surface of as-prepared loofah sponge was covered with coralloid silica nanoparticles to provide hierarchical nanoscale rough surfaces, which facilitated
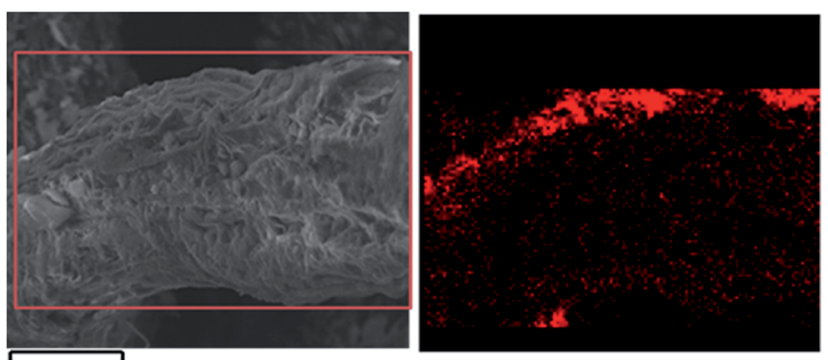

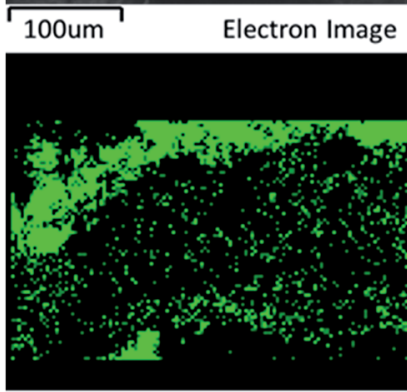

$\mathrm{O}$ Ka1

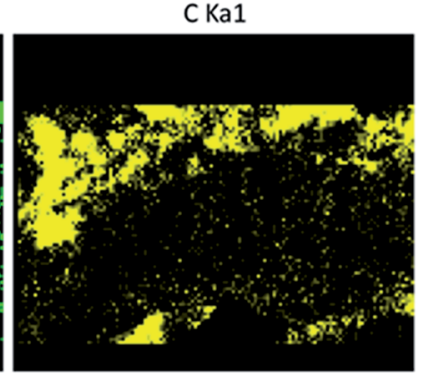

Si Ka1
Fig. 2 EDS elemental mapping images of the as-prepared loofah sponge.

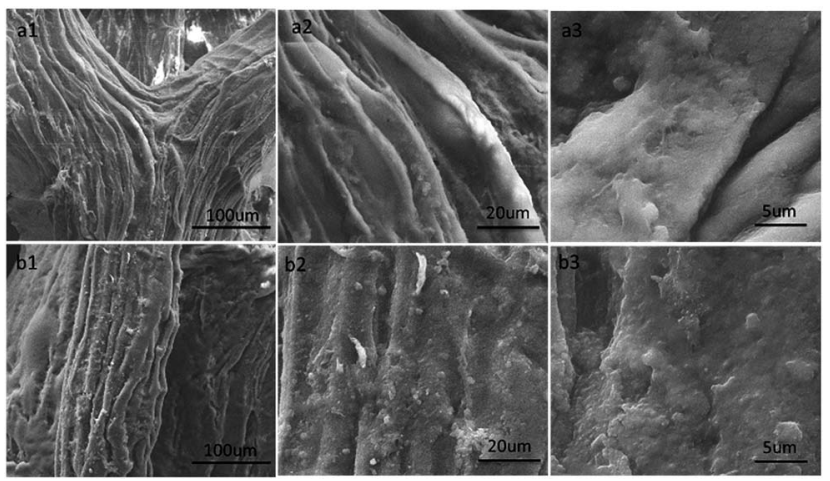

Fig. 3 FESEM images of the original loofah sponge (a) and the asprepared $\mathrm{SiO}_{2} @$ $\mathrm{WPU}$ modified loofah sponge (b).

the construction of super-wettability. In this system, the fused nano- $\mathrm{SiO}_{2}$ not only enhanced the surface roughness of the loofah sponge to construct 3D hierarchical rough surfaces, but also acted as a low surface energy material to replace the use of toxic low surface energy fluorocarbon substances, which endow the sponge with excellent superhydrophobic properties in an environmentally friendly approach.

The wettability of the as-prepared loofah sponge was investigated by water contact angle (WCA) measurements. As displayed in Fig. 4a, the as-prepared loofah sponge showed superhydrophobicity and superoleophilicity. When the oil droplet (oily red dyed chloroform) dropped onto the loofah sponge surface, it was absorbed quickly and disappeared completely, while the methylene blue dyed water droplets were repelled on the loofah surface and maintained their spherical shapes, exhibiting a water contact angle of $151.8^{\circ}$ (Fig. 4b). When being placed into water, the original loofah sponge rapidly absorbed water to expand and sank into water immediately (see Movie S1 of the ESI $\dagger$ ), while the as-prepared loofah sponge floated on the surface of the water due to its

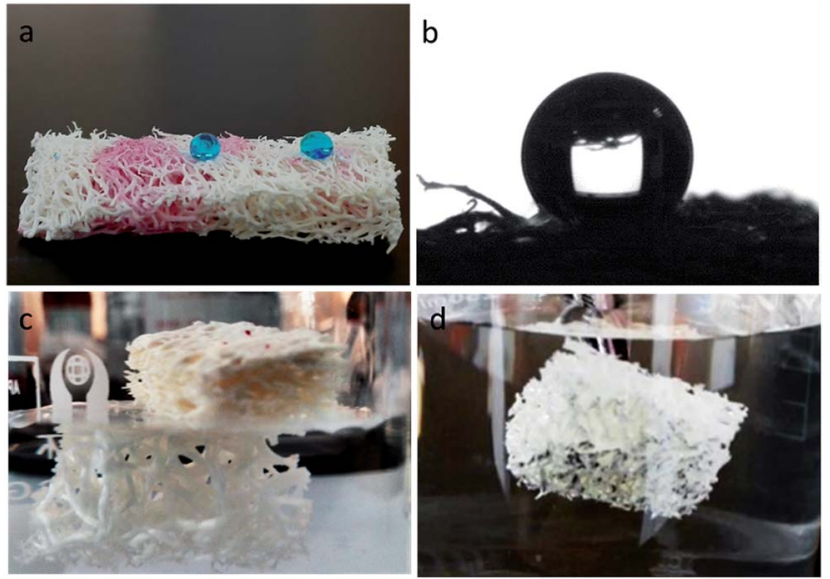

Fig. 4 Optical photos of (a) methylene blue dyed water and red oil dyed oil droplets on the as-prepared loofah surface, (b) the water contact angle in air, (c) original and as-prepared loofah after being placed in water, and (d) the as-prepared loofah immersed in water by an external force. 
superhydrophobicity (Fig. 4c). Moreover, the prepared loofah sponge appeared to have silver mirror-like surfaces when it was immersed into water by an external force (Fig. 4d). This nonwetting Cassie-Baxter behavior was due to a continuous air layer between the loofah sponge and water. ${ }^{55}$ The as-prepared loofah sponge also displayed a low adhesion to water. The water droplet (dyed with methylene blue) bounced and rolled off quickly from the loofah surface the moment it dropped on the surface (Fig. S1, see Movie S2 of the ESI $\dagger$ ).

The as-prepared loofah sponge showed excellent superhydrophobicity and superoleophilicity, which made it very promising as a versatile absorber material for removal of oil from water. As illustrated in Fig. 5a, the modified loofah sponge showed highly efficient selection to remove oil from water. Oily red dyed $n$-hexane spread on the water was rapidly and completely absorbed in a few seconds, leaving a transparent and clean region on the water surface due to the superhydrophobicity/superoleophilicity of the as-prepared loofah sponge (see Movie S3 of the ESI $\dagger$ ). The prepared sponge also showed excellent absorption selectivity toward organic solvents with higher densities than water by selectively absorbing the oily red dyed chloroform from under the water (Fig. 5b, see Movie S4 of the ESI $\dagger$ ). The rapid and complete absorption of organic solvents from water demonstrated the excellent oil/water separation capability of the as-prepared sponge.

Moreover, the as-prepared loofah sponge can separate oil microdroplets from a surfactant-free oil-in-water emulsion, which was prepared by mixing $n$-hexane in water $(\mathrm{v} / \mathrm{v}, 1 / 49)$ as shown in Fig. 6. Under intensive stirring for $0.5 \mathrm{~h}$, the asprepared loofah sponge was placed into the emulsion. Once touching the loofah sponge, emulsion droplets demulsified. The red oil droplets in the emulsion were immediately trapped and quickly absorbed by the loofah sponge. During the separation process, the loofah sponge slowly turned red and the emulsion gradually faded to transparent and clean in $1 \mathrm{~min}$, which suggests a highly efficient selective absorptive capacity of the as-prepared loofah sponge (Fig. 6a-d, see Movie S5 of the $\mathrm{ESI} \dagger$ ). Fig. $6 \mathrm{~b}$ and $\mathrm{c}$ show an obvious bright silver mirror layer on

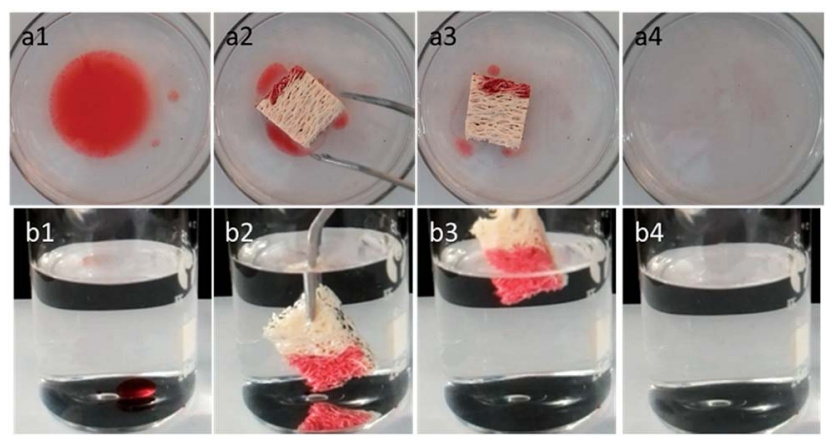

Fig. 5 Optical photos demonstrating the processes for oil-water separation of the as-prepared loofah: (a) sequential snapshots of removing a layer of oily red dyed $n$-hexane on top of water and (b) sequential snapshots of removing oily red dyed chloroform under water.

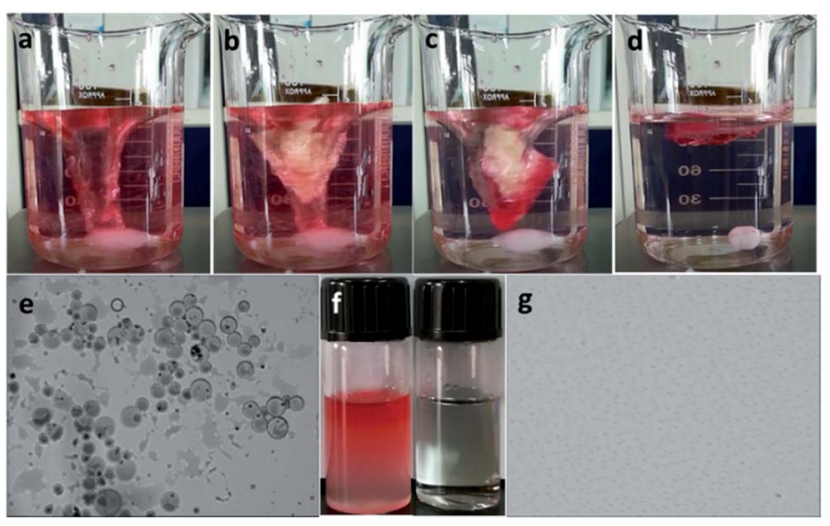

Fig. 6 Photographs of the selective collection of oil with a stirring process $(\mathrm{a}-\mathrm{d})$ and optical microscope images and digital photo of the separation results of the oil-in-water emulsion before and after oilwater separation $(\mathrm{e}-\mathrm{g})$.

the loofah surface when it was immersed into water, due to the reflectance of light at a continuous air layer between the loofah sponge and water. This suggested the loofah was in the nonwetting Cassie-Baxter state and still maintained excellent superhydrophobic properties. In addition, optical images of the $n$-hexane-in-water emulsion before and after oil separation further confirmed that the residual water was free of oil droplets (Fig. 6e-g), revealing a high separation efficiency for the separation of oil from surfactant-free oil-in-water emulsions.

The as-prepared loofah sponge also exhibited robust superhydrophobicity and excellent oil/water separation efficiency in harsh environments, such as immersed into $1 \mathrm{M} \mathrm{HCl}, 1 \mathrm{M}$ $\mathrm{NaOH}$, and saturated $\mathrm{NaCl}$ solutions, and hot water, respectively. The oil-water separation efficiency of the as-prepared loofah sponge for corrosive water/oil mixtures is depicted in Fig. 7. When directly immersed in a mixture of oily red dyed chloroform/n-hexane and $1 \mathrm{M} \mathrm{HCl}$ solution, the loofah sponge absorbed the oil and turned red rapidly due to the robust superhydrophobic/superoleophilic properties. The $\mathrm{HCl}$ water solution became clean and transparent with the volume nearly unchanged (Fig. 7a; Movie S6 in the ESI $\dagger$ ). Moreover, the loofah sponge also showed excellent oil/water separation ability in chloroform/n-hexane/1 M NaOH (Fig. 7b; Movie S7 in the ESI $\dagger$ ), chloroform/n-hexane/saturated NaCl (Fig. 7c; Movie S8 in the ESI $\dagger$ ), and hot water at higher than $95{ }^{\circ} \mathrm{C}$ (Fig. 7d; Movie S9 in the ESI $\dagger$ ) using the same method. And the loofah sponge still retained superhydrophobicity after 5 cycles as shown in Fig. S2. $\dagger$ These results demonstrated that the as-prepared loofah sponge maintained robust superhydrophobicity in harsh environments, which exhibited great potential in practical applications such as with ocean oily spills and wastewater from chemical industry.

As we all know, the construction of a superhydrophobic interface requires two elements: on the one hand was to construct a rough interface with hierarchical micro-nano structure on the hydrophobic surface, and the other hand was to modify the low surface energy material on the rough interface. ${ }^{56,57}$ In this work, the hydrophobic fused nano-SiO ${ }_{2}$ was 


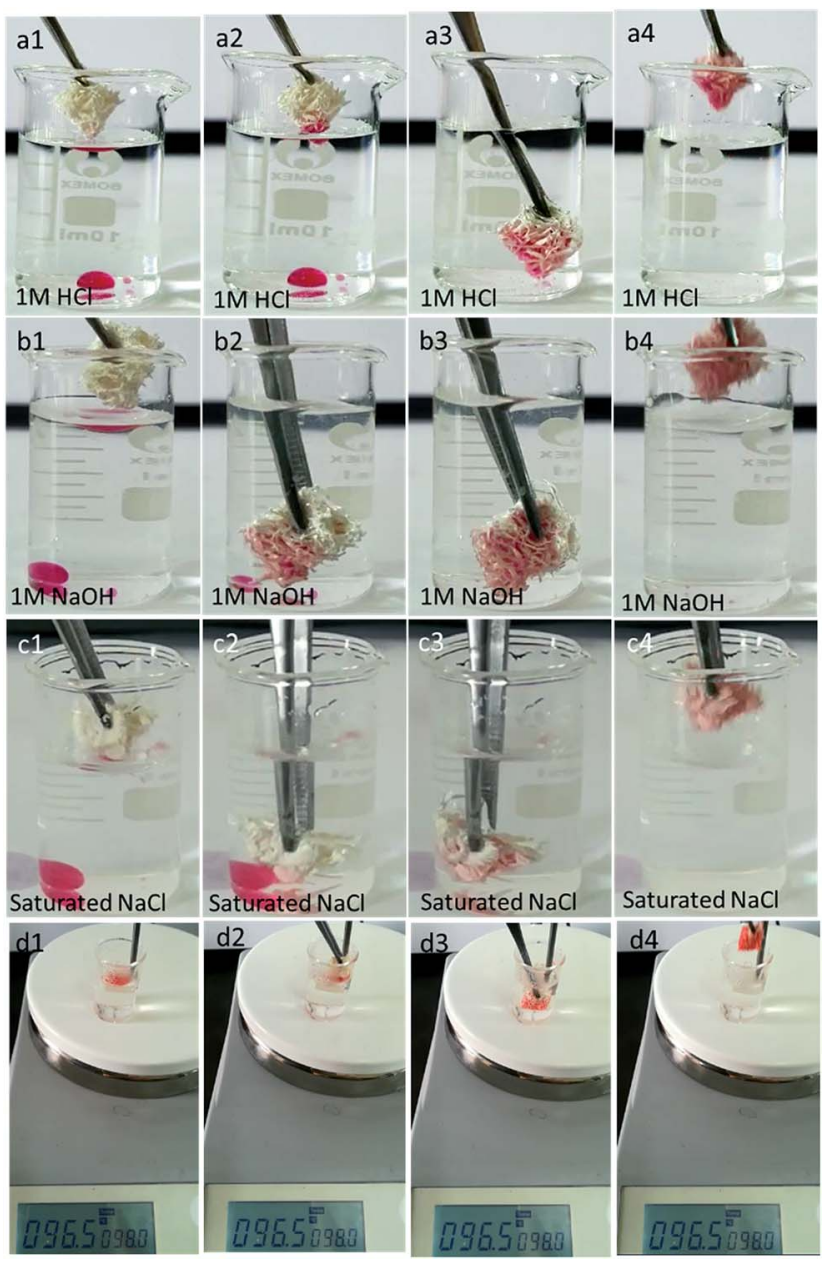

Fig. 7 The absorption process of the as-prepared loofah under harsh conditions by taking oily red dyed chloroform/n-hexane in corrosive solution mixtures as examples. (a) $1 \mathrm{M} \mathrm{HCl}$ solution, (b) $1 \mathrm{M} \mathrm{NaOH}$ solution, (c) saturated $\mathrm{NaCl}$ solution, and (d) oily red dyed kerosene in $95^{\circ} \mathrm{C}$ hot water.

introduced not only to provide a hierarchical micro-nano rough surface on the loofah sponge but also to act as a low-surfaceenergy compound material to endow the sponge with excellent superhydrophobic properties, which avoided the use of toxic, expensive fluorocarbon reagents. However, the fragile rough micro-nano structures of conventional superhydrophobic surfaces can be easily damaged under external mechanical forces or friction, which limits its large-scale application in practical industry. To solve this problem, the waterborne polyurea adhesive with $100 \%$ solid content was used as an adhesive to provide excellent adhesion, which can tightly bond $\mathrm{SiO}_{2}$ nanoparticles to the loofah sponge, and endow the sponge with superior chemical stability, abrasion resistance and robustness for oil-water separation in harsh environments.

In order to further check the robustness of the sponge against mechanical forces, the sandpaper-abrasion test and finger-wipe test were performed as shown in Fig. 8 and 9, respectively. In Fig. 8a, the as-prepared loofah sponge was
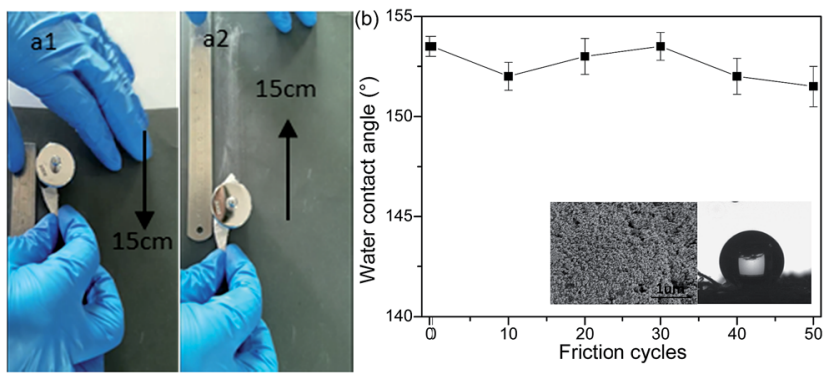

Fig. 8 Sandpaper-abrasion tests. (a1 and a2) One cycle of the sandpaper-abrasion test. (b) The variation of water CAs of the as-prepared sponge versus the number of abrasion cycles.

placed on the sandpaper under a weight of $100 \mathrm{~g}$ and moved back and forth with abrasion length of about $15 \mathrm{~cm}$. After a certain number of friction cycles, the sponge still preserved a good water repellency. Fig. 8b describes the water static contact angles after every tenth abrasion cycle and it can be seen that the water static contact angles were all higher than $150^{\circ}$, indicating that the loofah sponge possessed excellent superhydrophobicity and abrasion resistance (see Movie S10 in the ESI $\dagger$ ). The insets in Fig. $8 \mathrm{~b}$ are the SEM image and water static contact angle after 50 sandpaper-abrasion cycles for the loofah sponge surface. After 50 abrasion cycles, the loofah sponge surface still had a large amount of silica and the water contact angle was about $152^{\circ}$, which strongly indicated that the asprepared sponge retained robust superhydrophobicity due to the strong adhesion of the polyurea resin.

Fig. 9 demonstrates the finger-wipe test of loofah sponge. After several finger-wipes, the paint still remained on the sponge surface with no visible paint loss. The blue dyed water quickly fell off the surface of the sponge, which demonstrated the sponge surface retained superhydrophobicity (Fig. 9a1-a4, and see Movie S11 in the ESI $\dagger$ ). Fig. 9b depicts the water contact angle and oil/water separation efficiency of the as-prepared sponge during the 50 times finger-wipe test. As can be seen, the water contact angle of the sponge surface was still above $150^{\circ}$ after 50 abrasion cycles, which demonstrated the excellent mechanical abrasion resistance of the as-prepared superhydrophobic sponge. Meanwhile, the separation efficiency of the as-prepared sponge still remained above $95 \%$ after 50
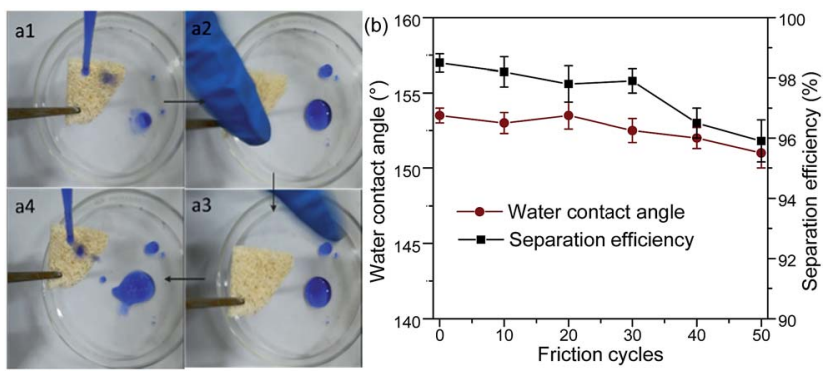

Fig. 9 Schematic illustration of the method of the finger-wipe test (a1 to a4). (b) The variation of water CAs and separation efficiency of the as-prepared sponge versus the number of finger-wipe cycles. 


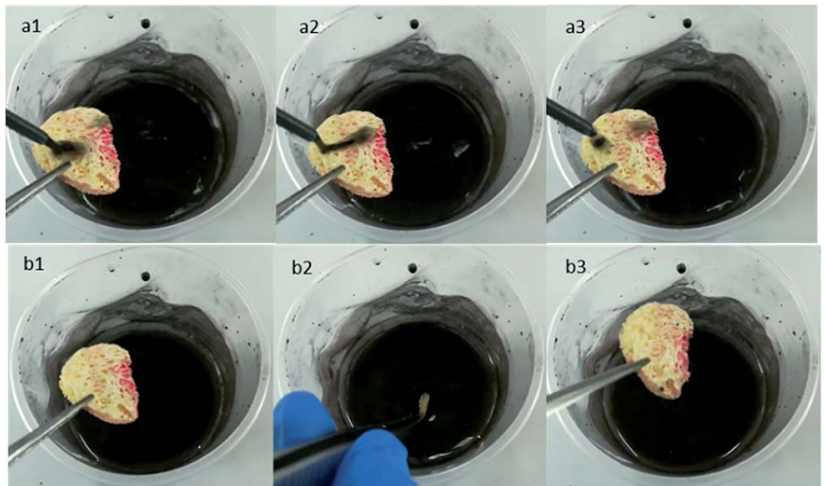

Fig. 10 Photographs of the self-cleaning property of the as-prepared sponge.

finger-wipes taking the $n$-hexane-water mixture as an example. These results indicated that the as-prepared superhydrophobic loofah sponge has excellent separation efficiency for the oilwater mixture against finger-wipe abrasion. The as-prepared superhydrophobic loofah sponge with strong abrasion resistance has great potential in practical industrial oil/water separation applications.

To our surprise, the superhydrophobic loofah sponge possessed antifouling and self-cleaning properties which were vital in practical oil/water separation applications since the fouling issues can seriously affect the absorption efficiency and cycle usage times of the absorbers. ${ }^{58}$ To observe the anti-fouling and self-cleaning properties, an abundant amount of manganese dioxide powder dispersed in water was used as a model contaminant. When the black sewage dripped onto the surface of the loofah sponge, the water droplets immediately rolled off the surface leaving a clean superhydrophobic surface (Fig. 10a1-a3). Moreover, after immersion in the black dirty solution, the surface of the as-prepared loofah sponge was almost free of black sewage indicating the excellent anti-fouling and self-cleaning performances (Fig. 10b1-b3 and see Movie S12 in the ESI + ). However, for the pristine loofah sponge there was a lot of black dirt accumulation on the pristine loofah sponge as shown in Fig. S3 and Movie S12 in the ESI. $\dagger$

\section{Conclusions}

In summary, we developed a facile, versatile and environmentally friendly strategy for construction of superhydrophobic loofah sponge coated with $\mathrm{WPU} / \mathrm{SiO}_{2}$ by the dip-coating method. The as-prepared loofah sponge exhibits excellent superhydrophobicity and efficient oil/water separation ability under a series of harsh conditions (such as $1 \mathrm{M} \mathrm{HCl}, 1 \mathrm{M} \mathrm{NaOH}$, saturated $\mathrm{NaCl}$ solution and hot water higher than $95^{\circ} \mathrm{C}$ ) due to the remarkably high chemical-stability. And the as-prepared loofah sponge also can effectively separate surfactant-free oilin-water emulsions and possesses excellent antifouling and self-cleaning properties against dirty contaminants. Moreover, the as-prepared loofah sponge has robust mechanical abrasion resistance, and shows excellent superhydrophobicity and oil/ water separation ability under strong abrasion using sandpaper and the finger-wipe test for more than 50 cycles. In addition, the whole process without involving poisonous chemically volatile reagents or expensive materials or sophisticated equipment can be easily scaled up. Therefore, the attractive features of the loofah sponge could make it an ideal adsorbent material for a range of applications including antifouling, self-cleaning, massive oil spill cleanup, and industrial oily wastewater purification.

\section{Conflicts of interest}

There are no conflicts to declare.

\section{Acknowledgements}

This work was supported by China Postdoctoral Science Foundation (2018M633054) and the High-Level Talent Start-Up Research Project of Foshan University (gg040945).

\section{Notes and references}

1 M. A. Shannon, P. W. Bohn, M. Elimelech, J. G. Georgiadis, B. J. Marinas and A. M. Mayes, Nature, 2008, 452, 301-310.

2 J. Ge, L. A. Shi, Y. C. Wang, H. Y. Zhao, H. B. Yao, Y. B. Zhu, Y. Zhang, H. W. Zhu, H. A. Wu and S. H. Yu, Nat. Nanotechnol., 2017, 12, 434-440.

3 J. E. Elliott and K. H. Elliott, Science, 2013, 340, 556-558.

4 Z. X. Xue, Y. Z. Cao, N. Liu, L. Feng and L. Jiang, J. Mater. Chem. A, 2014, 2, 2445-2460.

5 S. T. Wang, K. S. Liu, X. Yao and L. Jiang, Chem. Rev., 2015, 115, 8230-8293.

6 Z. R. Jiang, J. Ge, Y. X. Zhou, Z. Y. Wang, D. X. Chen, S. H. Yu and H. L. Jiang, NPG Asia Mater., 2016, 8, 253-260.

7 J. P. Chaudhary, S. K. Nataraj, A. Gogda and R. Meena, Green Chem., 2014, 16, 4552-4558.

8 Z. G. Xu, Y. Zhao, H. X. Wang, H. Zhou, C. X. Qin, X. G. Wang and T. Lin, ACS Appl. Mater. Interfaces, 2016, 8, 5661-5667.

9 G. Kwon, A. K. Kota, Y. Li, A. Sohani, J. M. Mabry and A. Tuteja, Adv. Mater., 2012, 24, 3666-3671.

10 Y. Lu, Y. Wang, L. Liu and W. Yuan, Environmental-friendly and magnetic/silanized ethyl cellulose sponges as effective and recyclable oil-absorption materials, Carbohydr. Polym., 2018, 173, 422-430.

11 F. Gao, L. P. Xu, Z. X. Xue, L. Feng, J. T. Peng, Y. Q. Wen, S. T. Wang and X. J. Zhang, Adv. Mater., 2014, 26, 1771.

12 A. A. Keller and V. Broje, Environ. Sci. Technol., 2006, 40, 7914-7918.

13 G. Wang, Z. X. Zeng, H. Wang, L. Zhang, X. D. Sun, Y. He, L. Y. Li, X. D. Wu, T. H. Ren and Q. J. Xue, ACS Appl. Mater. Interfaces, 2015, 7, 26184-26194.

14 M. G. Yu, Q. Wang, M. Zhang, Q. J. Deng and D. C. Chen, RSC Adv., 2017, 7, 39471-39479.

15 J. Li, D. M. Li, Y. X. Yang, J. P. Li, F. Zha and Z. Q. Lei, Green Chem., 2016, 18, 541-549.

16 J. Li, C. C. Xu, Y. Zhang, R. F. Wang, F. Zha and H. D. She, J. Mater. Chem. A, 2016, 4, 15546-15553. 
17 H. Zhang, Y. Q. Li, Z. X. Lu, L. H. Chen, L. L. Huang and M. Z. Fan, Sci. Rep., 2017, 7, 8428-8435.

18 C. R. Reshmi, S. P. Sundaran, A. Juraij and S. Athiyanathil, RSC Adv., 2017, 7, 2092-2102.

19 J. Li, R. M. Kang, X. H. Tang, H. D. She, Y. X. Yang and F. Zha, Nanoscale, 2016, 8, 7638-7645.

20 Q. Wang, M. G. Yu, G. X. Chen, Q. F. Chen and J. F. Tian, J. Mater. Sci., 2017, 52, 2549-2559.

21 B. S. Al-anzi and O. C. Siang, RSC Adv., 2017, 7, 20981-20994.

22 L. Feng, Z. Zhang, Z. Mai, Y. Ma, B. Liu, L. Jiang and D. Zhu, Angew. Chem., Int. Ed., 2004, 43, 2012-2014.

23 L. J. Li, L. Liu, J. L. Lei, J. X. He, N. B. Li and F. S. Pan, J. Mater. Chem. A, 2016, 4, 12334-12340.

24 W. B. Zhang, Y. Z. Zhu, X. Liu, D. Wang, J. Y. Li, L. Jiang and J. Jin, Angew. Chem., Int. Ed., 2014, 53, 856-860.

25 J. Li, L. Yan, X. H. Tang, H. Feng, D. H. Hu and F. Zha, Adv. Mater. Interfaces, 2016, 3, 1500770-1500777.

26 R. K. Gupta, G. J. Dunderdale, M. W. England and A. Hozumi, J. Mater. Chem. A, 2017, 5, 16025-16058.

27 Y. Lu, S. Sathasivam, J. L. Song, C. R. Crick, C. J. Carmalt and I. P. Parkin, Science, 2015, 347, 1132-1135.

28 M. E. Buck, S. C. Schwartz and D. M. Lynn, Chem. Mater., 2010, 22, 6319-6327.

29 C. H. Su, Y. Q. Xu, F. Gong, F. S. Wang and C. F. Li, Soft Matter, 2010, 6, 6068-6071.

30 B. Y. Chen, J. H. Qiu, E. Sakai, N. Kanazawa, R. L. Liang and H. X. Feng, ACS Appl. Mater. Interfaces, 2016, 8, 17659-17667.

31 L. W. Chen, Z. G. Guo and W. M. Liu, ACS Appl. Mater. Interfaces, 2016, 8, 27188-27198.

32 W. S. Y. Wong, Z. H. Stachurski, D. R. Nisbet and A. Tricoli, ACS Appl. Mater. Interfaces, 2016, 8, 13615-13623.

33 J. Zhao, Y. Li, J. L. Sheng, X. F. Wang, L. F. Liu, J. Y. Yu and B. Ding, ACS Appl. Mater. Interfaces, 2017, 9, 29302-29310.

34 H. M. Jang, H. S. Lee, K. S. Lee and D. R. Kim, ACS Appl. Mater. Interfaces, 2017, 9, 9213-9220.

35 L. Zhang, H. Li, X. Lai, X. Su, T. Liang and X. Zeng, Chem. Eng. J., 2017, 316, 736-743.

36 X. Su, H. Li, X. Lai, L. Zhang, J. Wang, X. Liao and X. Zeng, ACS Appl. Mater. Interfaces, 2017, 9, 28089-28099.

37 X. Su, H. Li, X. Lai, L. Zhang, T. Liang, Y. Feng and X. Zeng, ACS Appl. Mater. Interfaces, 2017, 9, 3131-3141.

38 X. Su, H. Li, X. Lai, L. Zhang, X. Liao, J. Wang, Z. Chen, J. He and X. Zeng, ACS Appl. Mater. Interfaces, 2018, 10, 4213-4221.
39 Y. Yang, Y. H. Deng, Z. Tong and C. Y. Wang, ACS Sustainable Chem. Eng., 2014, 2, 1729-1733.

40 Q. Wang, M. G. Yu, G. X. Chen, Q. F. Chen and J. F. Tian, BioResources, 2017, 12, 643-654.

41 H. Z. Sai, R. Fu, L. Xing, J. H. Xiang, Z. Y. Li, F. Li and T. Zhang, ACS Appl. Mater. Interfaces, 2015, 7, 7373-7381.

42 A. Mulyadi, Z. Zhang and Y. L. Deng, ACS Appl. Mater. Interfaces, 2016, 8, 2732-2740.

43 Z. Zhang, G. Sebe, D. Rentsch, T. Zimmermann and P. Tingaut, Chem. Mater., 2014, 26, 2659-2668.

44 S. Wang, X. W. Peng, L. X. Zhong, J. W. Tan, S. S. Jing, X. F. Cao, W. Chen, C. F. Liu and R. C. Sun, J. Mater. Chem. A, 2015, 3, 8772-8781.

45 J. Li, Z. Zhao, D. Li, H. Tian, F. Zha, H. Feng and L. Guo, Nanoscale, 2017, 9, 13610-13617.

46 S. Zhou, P. P. Liu, M. Wang, H. Zhao, J. Yang and F. Xu, ACS Sustainable Chem. Eng., 2016, 4, 6409-6416.

47 X. D. Tang, C. L. Shen, W. J. Zhu, S. Zhang, Y. F. Xu, Y. Q. Yang, M. Gao and F. Y. Dong, RSC Adv., 2017, 7, 30495-30499.

48 N. T. Cervin, C. Aulin, P. T. Larsson and L. Wagberg, Cellulose, 2012, 19, 401-410.

49 G. Wang, Y. He, H. Wang, L. Zhang, Q. Y. Yu, S. S. Peng, X. D. Wu, T. H. Ren, Z. X. Zeng and Q. J. Xue, Green Chem., 2015, 17, 3093-3099.

50 J. X. Jiang, Q. H. Zhang, X. L. Zhan and F. Q. Chen, ACS Sustainable Chem. Eng., 2017, 5, 10307-10316.

51 J. P. Chaudhary, N. Vadodariya, S. K. Nataraj and R. Meena, ACS Appl. Mater. Interfaces, 2015, 7, 24957-24962.

52 Y. B. Peng and Z. G. Guo, J. Mater. Chem. A, 2016, 4, 1574915770.

53 C. Schlaich, L. X. Yu, L. C. Camacho, Q. Wei and R. Haag, Polym. Chem., 2016, 7, 7446-7454.

54 Q. L. Fu, F. Ansari, Q. Zhou and L. A. Berglund, ACS Nano, 2018, 12, 2222-2230.

55 B. Su, Y. Tian and L. Jiang, J. Am. Chem. Soc., 2016, 138, 17271748.

56 X. F. Gao and L. Jiang, Nature, 2004, 432, 36.

57 L. Feng, S. H. Li, Y. S. Li, H. J. Li, L. J. Zhang, J. Zhai, Y. L. Song, B. Q. Liu, L. Jiang and D. B. Zhu, Adv. Mater., 2002, 14, 1857-1860.

58 C. Y. Cao, M. Z. Ge, J. Y. Huang, S. H. Li, S. Deng, S. N. Zhang, Z. Chen, K. Q. Zhang, S. S. Al-Deyab and Y. K. Lai, J. Mater. Chem. A, 2016, 4, 12179-12187. 